\title{
SURGERY
}

\section{Remission of type 2 diabetes mellitus after bariatric surgery-is it durable?}

Two teams of researchers have evaluated the long-term effects of bariatric surgery on remission of type 2 diabetes mellitus (T2DM) in adults with severe obesity $\left(\mathrm{BMI} \geq 35 \mathrm{~kg} / \mathrm{m}^{2}\right)$.

\section{1 ...the rate of remission of T2DM 6 years after surgery was $62 \% \ldots 77$}

In the first study, Jiménez et al. assessed the durability of T2DM remission after bariatric surgery and the predictors of T2DM remission and recurrence after surgery. The researchers followed up 153 individuals with T2DM and severe obesity for $35.4 \pm 13.5$ months after they underwent sleeve gastrectomy or Roux-en-Y gastric bypass surgery. The rate of remission of T2DM was $66 \%$ for participants at their last follow-up visit, and was comparable for the two surgical techniques. Predictors of a lack of remission of T2DM after surgery included a long duration of T2DM, high $\mathrm{HbA}_{1 \mathrm{c}}$ level, insulin treatment at study entry and low excess weight loss at last follow-up.
Predictors of recurrence of T2DM after surgery included insulin use and weight regain after remission.

In the second study, Adams and co-workers evaluated the health benefits of Roux-en-Y gastric bypass surgery, including remission of T2DM. In 418 severely obese individuals who underwent such surgery, the rate of remission of T2DM 6 years after surgery was $62 \%$, compared with $8 \%$ in 417 severely obese individuals who sought but did not have surgery and 6\% in 312 individuals with severe obesity who had not sought bariatric surgery. Bariatric surgery was also associated with significant weight loss and improved metabolic and cardiovascular risk profiles after 6 years.

Importantly, the new data shed light on strategies to maximize long-term remission rates of T2DM after bariatric surgery. "Our data would support that bariatric surgery for the severely obese with T2DM should be considered early as a therapeutic option, rather than being held back as a last resort," comments Josep Vidal, senior author of the study by Jiménez et al. "Weight loss was a

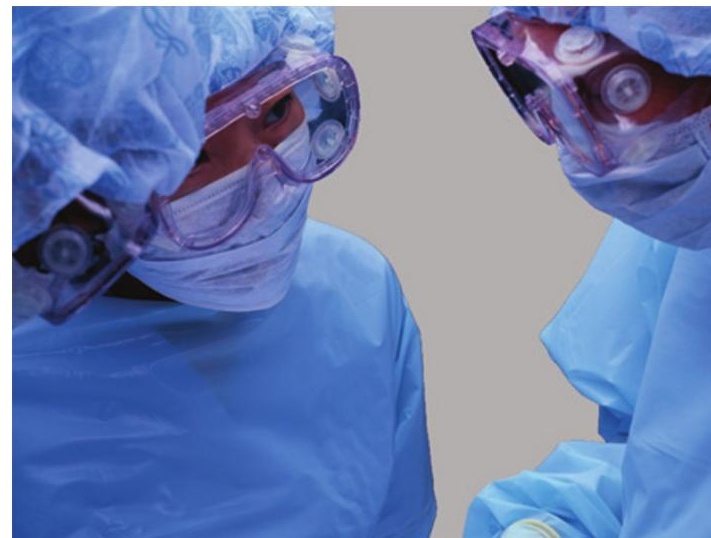

significant predictor of remission, and weight gain independently predicted diabetes recurrence. Thus, efforts should be made to help the bariatric surgery patient to keep their weight off."

Carol Wilson

Original articles Jiménez, A. et al. Long-term effects of sleeve gastrectomy and Roux-en-Y gastric bypass surgery on type 2 diabetes mellitus in morbidly obese subjects. Ann. Surg. doi:10.1097/SLA.0b013e318262ee6b | Adams, T. D. et al. Health benefits of gastric bypass surgery after 6 years. JAMA 308, 1122-1131 (2012) 